\title{
Agrobc: Gestión Descentralizada de Cadenas de Valor Agrícolas Usando Tecnología Blockchain
}

\section{AgroBC: decentralized management of agricultural value chains using Blockchain technology}

\author{
Mosquera, Jean $^{1}$ \\ Universidad Técnica Particular de Loja, Ecuador \\ https://orcid.org/0000-0002-2215-4081 \\ Piedra, Nelson ${ }^{2}$ \\ Knowledge Based Systems Research Group - UTPL, Ecuador \\ https://orcid.org/0000-0003-1067-8707
}

Recibido:25-09-2020

Aceptado: 21-12-2020

\section{Cita Recomendada}

Mosquera, J. \& Piedra, N. (2020). AGROBC: Gestión descentralizada de cadenas de valor agrícolas usando tecnología Blockchain. Hamut'ay, 7 (3), 98-105

http://dx.doi.org/10.21503/hamu.v7i3.2201

\begin{abstract}
Resumen
La tecnología blockchain gracias a sus características innatas que son la transparencia, descentralización, trazabilidad, entre otras, durante los últimos años está siendo ampliamente usada en diversos sectores entre estos el sector agrícola. Además, en el mundo se pueden encontrar diversas soluciones enfocadas en la educación, finanzas, transporte y diversas industrias entre estas la industria agrícola. Mediante el presente trabajo se busca proponer una aplicación descentralizada empleando la tecnología blockchain para lograr la transparencia en el sector agrícola, además de buscar el permitir la gestión descentralizada de la cadena de suministros de forma descentralizada, así como el proceso seguido para el desarrollo de una aplicación descentralizada para la gestión de la cadena de suministros. Como resultados se presenta la aplicación descentralizada, la cual demuestra que es posible lograr transparentar la cadena de suministros del sector agrícola, convirtiendo así a este sector aún más atractivo ante los consumidores, además de permitir mejorar la gestión de la cadena de suministros, que ya de por si su naturaleza es compleja. Finalmente, los autores concluyen que es posible el empleo de la tecnología blockchain en el ámbito agrícola con el objetivo de lograr la transparencia de este sector productivo, también discuten aspectos adicionales que deben ser considerados y se ha demostrado el potencial de la tecnología blockchain en el desarrollo de soluciones en diversas industrias.
\end{abstract}

Palabras Clave: Blockchain, Agricultura, Gestión

\footnotetext{
1. Estudiante de Sistemas Informáticos y Computación en la Universidad Técnica Particular de Loja, con experiencia en el desarrollo de aplicaciones móviles y web, Knowledge Graphs, Blockchain e inteligencia artificial. jpmosquera1@utpl.edu.ec

2. Docente-investigador en el Departamento de Ciencias de la Computación en la UTPL. Ingeniero en Informática y en Administración de Empresas. $\mathrm{PhD}$ en Software y Sistemas en Universidad Politécnica de Madrid. Actualmente es Director de Transferencia de conocimiento en el Vicerrectorado de Investigación de UTPL. nopiedra@utpl.edu.ec
} 


\begin{abstract}
Blockchain technology, thanks to its innate characteristics that are transparency, decentralization, traceability, among others, in recent years is being widely used in various sectors, including the agricultural sector. In addition, in the world you can find various solutions focused on education, finance, transportation and various industries, including the agricultural industry. The present work seeks to propose a decentralized application using blockchain technology to achieve transparency in the agricultural sector, in addition to allowing decentralized management of the supply chain in a decentralized way. Thus, in this work the process followed for the development of a decentralized application for the management of the supply chain is presented. As results, the decentralized application is presented, which shows that it is possible to achieve transparency in the supply chain of the agricultural sector, thus making this sector even more attractive to consumers, in addition to allowing to improve the management of the supply chain that already exists. if its nature is complex. Finally, the authors conclude that the use of blockchain technology in the agricultural field is possible with the aim of achieving transparency in this productive sector, they also discuss additional aspects that must be considered and have demonstrated the potential of blockchain technology in development. of solutions in various industries.
\end{abstract}

Key words: Blockchain, Agricultural, Management

\section{Introducción}

El sector agrícola en la actualidad está siendo de interés de muchos actores, los cuales han buscado lograr la innovación e implantación tecnológica con el único fin de lograr mejorar la producción, rentabilidad, y sobre todo proveer confianza a los consumidores finales y en si beneficiar a todos los actores que hacen posible la producción alimenticia del mundo (Food and Agriculture Organization of the United Nations, 2019).

Según la Organización de las Naciones Unidas para la Alimentación y la Agricultura (FAO) la agricultura tiene gran importancia en la economía de todos los países del mundo, más aún en los países en desarrollo. Además de ser la base de la seguridad alimentaria tema de gran interés en el mundo en la actualidad al ser parte de la Agenda 2030 para el desarrollo sostenible (Food and Agriculture Organization of the United Nations, 2020).

Con el avance de la tecnología ha surgido el interés por la gestión y estructuración de la información generada en diversos aspectos o ámbitos, entre ellos la cultura, industria entre otros (Piedra \& Mosquera Arévalo, 2020). La tecnología blockchain es un ledger o libro mayor que es casi imposible de falsificar debido a que los registros contenidos en este se encuentran enlazados, bajo cifrado y replicados a lo largo de una red de nodos con el objetivo de lograr la integridad transaccional (Laurence, 2019; Prieto \& Ferretti, 2020).

En la actualidad el uso de tecnologías emergentes tales como Blockchain (BC) y Distributed Ledger, entre otras han logrado mucha connotación en varias industrias, entre las cuales se destacan el Internet de las cosas (IoT), educación, ámbito bancario, y con gran impacto en la industria productiva y manufacturera en la cadena de producción y suministros (Borah et al., 2020; Dhillon et al., 2017).

Esta investigación se centra en el desarrollo de un prototipo que permita la gestión descentralizada de la cadena de suministros del sector agrícola empleando tecnología blockchain, para de este modo lograr transparencia, seguridad y más ventajas que trae consigo la aplicación de la tecnología blockchain, lo que genera está en muchos beneficios para el sector Agrícola.

Para el desarrollo del prototipo se propone una metodología, la cual comprende desde el análisis de la cadena de valor hasta la ejecución de pruebas de la aplicación desarrollada para gestionar dicha cadena de valor. Es importante destacar que las fases que se proponen son esenciales y primordiales para lograr el desarrollo de una solución efectiva que se acople a la industria de 
interés. Como aproximación se propone emplear la plataforma blockchain Ethereum debido a que en la actualidad dicha plataforma es ampliamente empleada en el desarrollo de soluciones blockchain y tecnologías auxiliares necesarias que son descritas en la arquitectura propuesta para la solución.

\section{Método}

La tecnología blockchain tiene un sinfín de aplicaciones en el sector agrícola de este modo mediante la revisión de literatura se busca contrastar diversos aspectos relacionados con tecnologías, y aproximaciones similares para enriquecer la propuesta sugerida por la presente investigación. Para el desarrollo de la revisión de literatura se ha optado por el empleo de una adaptación de la metodología propuesta por Okoli y Schabram (2010) en la que se abordan diversos aspectos, desde la definición de preguntas hasta la discusión de los trabajos. Así la figura 1 detalla de forma resumida la metodología adaptada.

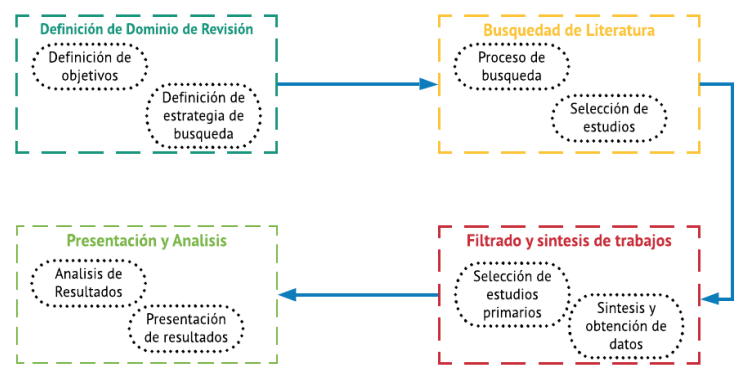

Figura 1. Metodología seguida para la revisión de literatura Fuente: Elaboración propia (2020)

\section{Definición de Dominio de Revisión}

Entre los objetivos que persigue la revisión de literatura es definir las tecnologías más empleadas y sugeridas por autores que se encuentran trabajando en el ámbito de acción de esta investigación. Además de buscar y definir varios aspectos concluidos en dichos trabajos. En la estrategia de búsqueda se ha definido considerar los trabajos comprendidos entre los 4 últimos años (20162019) relacionados con blockchain y el ámbito agrícola en 4 bases de datos científicas de importancia, las cuales son: SpringerLink, Scopus, ScienceDirect (Elsevier), IEEE Xplore.

\section{Búsqueda de literatura.}

Previo a la ejecución de la búsqueda en las bases de datos previamente indicadas se han definido las cadenas de búsqueda asociadas a los términos o conceptos que comprendan el ámbito de acción de la investigación, de este modo en la tabla 1 se detalla las cadenas de búsqueda.

Tabla 1. Cadenas de búsqueda definidas.

\begin{tabular}{|c|c|}
\hline Base de datos & Cadena de búsqueda \\
\hline SpringerLink & $\begin{array}{l}\text { ((agriculture OR agricultural) OR food) and (blockchain } \\
\text { AND supply chain) AND traceability }\end{array}$ \\
\hline Scopus & $\begin{array}{l}\text { TITLE-ABS-KEY ((((agriculture OR agricultural) OR food) } \\
\text { AND (blockchain AND supply AND chain) AND } \\
\text { traceability }))\end{array}$ \\
\hline $\begin{array}{l}\text { ScienceDirect } \\
\text { (Elsevier) }\end{array}$ & $\begin{array}{l}\text { (((agriculture OR agricultural) OR food) AND (blockchain } \\
\text { AND supply chain) AND traceability) }\end{array}$ \\
\hline IEEE & $\begin{array}{c}\text { (((agriculture OR agricultural) OR food) AND (blockchain } \\
\text { AND supply chain) AND traceability) }\end{array}$ \\
\hline
\end{tabular}

Con las cadenas de búsqueda definidas se realizó el proceso de obtención de resultados en las bases de datos, lo cual arrojo los siguientes resultados: En SpringerLink dieron como resultado 151 trabajos, en IEEE Xplore 19 trabajos, en Science direct 190 trabajos, y en Scopus un total de 52 trabajos.

\section{Filtrado y síntesis de la literatura}

En esta fase de los trabajos obtenidos como resultado se seleccionan los trabajos primarios, los cuales serán objeto de análisis. De este modo para esta selección se consideró el análisis del título, el abstract y el texto completo del trabajo. Como se detalla en la figura 2 , en la que se puede contrastar todo el proceso seguido de forma resumida para la obtención de los trabajos primarios tomando en cuenta los objetivos previamente definidos y en las que se encuentra centrada la revisión de literatura. 


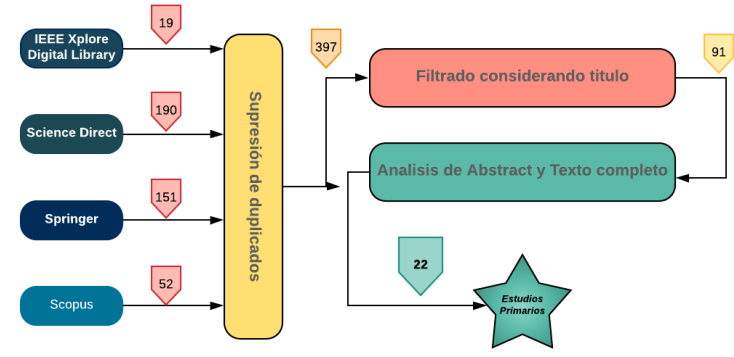

Figura 2. Proceso de filtrado y selección de trabajos primarios Fuente: Elaboración propia (2020)

Los 22 trabajos definidos como primarios comprenden trabajos que abordan el desarrollo de aplicaciones desarrolladas para el sector agrícola entre los cuales se destacan sistemas para la cadena de suministros agrícola, soluciones para la certificación de proveniencia, soluciones de trading de alimentos, y sistemas de trazabilidad de alimentos.

Presentación y análisis.

La mayor parte de aplicaciones blockchain en el ámbito agrícola abordadas en los trabajos primarios corresponden a sistemas para la cadena de suministros agrícola así 14 de los 22 trabajos abordan aplicaciones de este tipo, mientras que 6 trabajos están enfocados en soluciones para lograr la trazabilidad de alimentos y los trabajos que restan están enfocados a soluciones para el trading y certificación.

En lo concerniente a tecnologías se pudo visualizar que existe mayor preferencia hacia la plataforma Ethereum al momento del desarrollo de soluciones blockchain. Sin dejar de lado que existen distintas alternativas adicionales como son Hyperledger Fabric, Hyperledger Sawthooth, BigChainDB.

Otro aspecto que se ha considerado esencial tomar en cuenta, es el alcance de los trabajos primarios. De este modo se seleccionaron los trabajos que consistían en el desarrollo integral de una aplicación descentralizada, los cuales constituían un total de 12.
Tabla 2. Trabajos primarios según su alcance

\begin{tabular}{|c|c|c|}
\hline Alcance & Descripción & Artículos \\
\hline $\begin{array}{c}\text { Solución Integral } \\
\qquad(\mathrm{n}=12)\end{array}$ & $\begin{array}{l}\text { Trabajos en los que se } \\
\text { presentan Arquitectura, } \\
\text { implementación, } \\
\text { ejecución de pruebas de } \\
\text { desempeño } \\
\text { (Performance). }\end{array}$ & $\begin{array}{l}\text { Caro (Caro et al., 2018), } \\
\text { Salah (Salah et al., 2019), } \\
\text { Lin (Lin et al., 2019), } \\
\text { Malik (Malik et al., 2018), } \\
\text { Mao(Mao et al., 2019), } \\
\text { Branco(Branco et al., 2019), } \\
\text { Huang(Huang et al., 2019), } \\
\text { Borrero (Borrero, 2019), } \\
\text { Arena(Arena et al., 2019), } \\
\text { Baralla(Baralla et al., 2019), } \\
\text { Bordel(Bordel et al., 2018), }\end{array}$ \\
\hline
\end{tabular}

Fuente: Elaboración propia (2020)

Además, según el análisis de los trabajos relacionados se pudo observar un gran interés por la aplicación de la tecnología blockchain en el sector agrícola, agropecuario y alimentario. La revisión de literatura además permitió establecer una guía para la selección de aspectos relacionados con la tecnología como son las plataformas blockchain en la que la plataforma Ethereum es la más preferida al momento del desarrollo de soluciones enfocadas en la industria agrícola.

\section{Metodología del prototipo}

Para el desarrollo del prototipo se propone una metodología fundamentada en 4 fases las cuales pueden ser vistas en la figura 3. Es importante destacar que a modo ilustrativo se ha elegido una industria agrícola específica, no obstante, se debe mencionar que la metodología propuesta se adapta a cualquier otra industria agrícola.

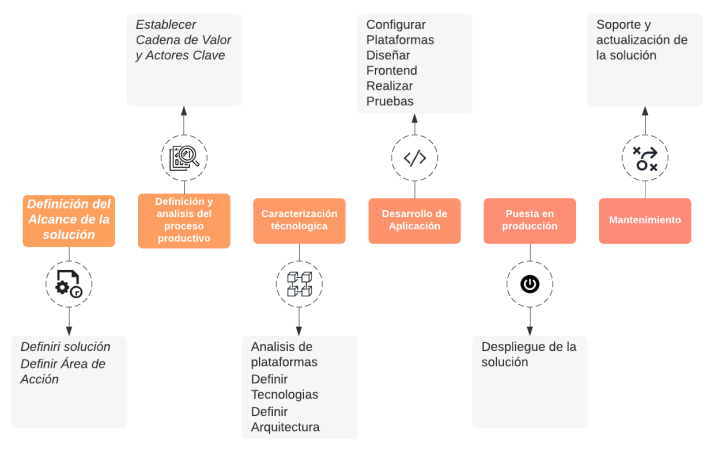

Figura 3 Metodología para el desarrollo de una solución blockchain para el ámbito Agrícola. Fuente: Elaboración propia (2020) 
Es importante añadir que varios autores y organizaciones como por ejemplo GS1 coinciden en la importancia del análisis previo de la cadena de valor de la industria en la que se desea implantar la solución.

La metodología que se propone, aparte de ser fácil de comprender abarca todos los aspectos clave para el desarrollo de una solución en función a las necesidades reales de la industria.

\section{Procedimiento}

Presentada la metodología que conducirá el desarrollo del prototipo se describe el proceso seguido en cada fase de forma descriptiva, como previamente se ilustró se ha tomado una industria específica, pero esta puede adaptarse y acoplarse para cualquier otra industria agrícola, agropecuaria y/o alimenticia.

\section{Definición de alcance de la solución}

En esta fase entre los factores que se establecen se encuentran el dominio de trabajo y la solución que se desarrollará, de este modo se propone trabajar con la industria cafetalera, una industria con gran impacto a nivel de la provincia de Loja, cuyo café es reconocido a nivel de Ecuador y del extranjero. La solución que se desarrollará es una aplicación para permitir la gestión de la cadena de valor de esta industria y además lograr la transparencia empleando tecnología blockchain.

\section{Definición y análisis del proceso productivo}

Una vez establecido el campo de acción en esta etapa se analiza el proceso productivo con el objetivo de definir la cadena de valor, es importante destacar que esto dependería específicamente de la industria y en si del proceso productivo seguido por el actor o actores que forman parte de este. Entre fases genéricas en el ámbito agrícola se encuentran la producción, procesamiento, distribución, comercialización (Viere et al., 2011).

Para este proceso se ha revisado y utilizado de varios trabajos que abordan el análisis de la industria, además de contrastar esto mediante en- trevistas a expertos logrando de este modo definir la cadena de valor y los actores que forman parte de esta, lo cual se encuentra ilustrado en la figura 4, en la que se puede observar la cadena de valor, la información que se genera en cada fase, y los actores que forman parte de esta o realizan el proceso productivo, para de este modo lograr una mejor comprensión de quienes serán parte del proyecto y en si harán uso de la solución. Este análisis previo es de mucha ayuda puesto que , permite conocer de forma precisa la situación real que se da en lo referente al registro de información en cada fase.

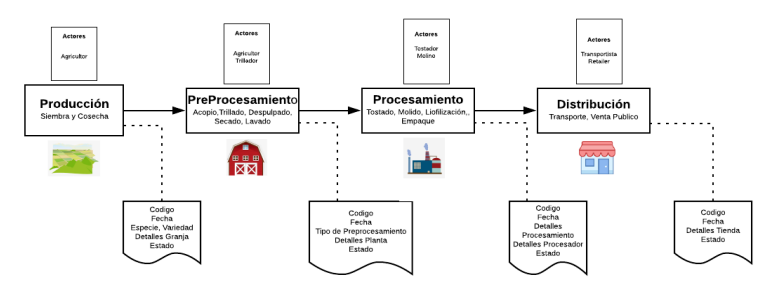

Figura 4. Cadena de valor

Fuente: Elaboración propia (2020)

\section{Análisis tecnológico y definición de arquitectura}

Una vez analizado el proceso productivo y definida la cadena de valor con los insumos adicionales, es posible efectuar el análisis tecnológico, es importante destacar que una aplicación descentralizada está conformada de dos componentes principales que son la parte relacionada con la tecnología blockchain y el frontend de la aplicación, es decir, lo que permitirá o hará posible el uso de la aplicación a los actores.

En lo relacionado al análisis de tecnología blockchain se tomó en cuenta la recomendación realizada por Dhillon et al. (2017) y Takyar (2020) que indican varios aspectos para elegir una plataforma Blockchain, en la tabla 3 se presenta las comparaciones realizadas entre las plataformas más empleadas en desarrollo de soluciones para el sector agrícola. 
Tabla 3 Comparación de plataformas Blockchain

\begin{tabular}{cccc}
\hline Característica & Ethereum & $\begin{array}{c}\text { Hyperledger } \\
\text { Fabric }\end{array}$ & $\begin{array}{c}\text { Hyperledger } \\
\text { Sawtooth }\end{array}$ \\
\hline $\begin{array}{c}\text { Contratos } \\
\text { Inteligentes } \\
\text { Lenguajes } \\
\text { Compatibles }\end{array}$ & Si (Solidity) & Si (Java, Golang) & Si (C++, Golang) \\
& $\begin{array}{c}\text { Java, Python, } \\
\text { Just, .NET, Delphi }\end{array}$ & $\begin{array}{c}\text { Go, Java, Javascript, } \\
\text { Python }\end{array}$ & $\begin{array}{c}\text { Go,, Java, Javascript, } \\
\text { Python, Rust, Swift }\end{array}$ \\
\hline Tipo de blockchain & Publica & Consorcio & Consorcio \\
\hline Escalabidad & Permitida & Permitida & Permitida \\
\hline & &
\end{tabular}

Ethereum es la plataforma blockchain que más se adecua para el desarrollo de la solución, puesto que permite escalabilidad a lo largo del tiempo y además de ser un tipo de blockchain pública, lo que es de gran interés al querer buscar la transparencia.

Para la definición del framework a emplearse para el frontend se ha tomado en cuenta una encuesta realizada durante todos los años por la empresa Fluence Labs, en la que destaca React.js como el framework más empleado en el desarrollo de soluciones blockchain, entre algunas de sus cualidades se encuentran la adaptabilidad según el dispositivo desde donde se ingrese a la aplicación. Importante mencionar que las aplicaciones descentralizadas se constituyen en aplicaciones web, sin dejar de lado que los esfuerzos apuntan a habilitar el desarrollo de aplicaciones móviles descentralizadas (Ponomarev, 2020).

(Mosquera \& Piedra, 2020) presentan una arquitectura para el desarrollo de una aplicación descentralizada en la industria del café, en la cual los autores emplean Ethereum como plataforma blockchain y react para el desarrollo del frontend de la aplicación, además los autores distinguen las distintas herramientas y servicios empleadas tanto en la fase de desarrollo de la solución como en la etapa de funcionamiento o de producción de la aplicación descentralizada.

\section{Desarrollo de la aplicación}

En esta fase se efectúa el desarrollo de la solución, es decir se codifican los contratos inteligentes, además de realizar la configuración de la plataforma blockchain de la cual se hará uso.

Es importante destacar que se empleará un es- quema de roles para controlar quienes hacen uso de la aplicación, además se busca que la información prevista sea verídica. El contrato SupplyChain es el contrato que hace posible el registro de la información, este contrato contiene todas las funciones que permiten el registro en la cadena de bloques de la información generada. En esta fase también se realiza el desarrollo del frontend de la solución, el cual se presentará en la sección de resultados. Una vez concluidos estos dos desarrollos se ejecutan pruebas para establecer el correcto funcionamiento de la solución, en caso de presentarse algún fallo es posible de remediarse antes de que la solución este en un ambiente de producción.

\section{Puesta en producción y Mantenimiento}

Una vez desarrolladas con éxito las pruebas de la solución se debe desplegar la solución para que esta sea empleada por los actores. Es relevante destacar que esto conllevaría un costo, es decir, al momento de desplegar los contratos inteligentes en la red principal de Ethereum, con fines de simulación se ha hecho uso de redes de prueba mediante las cuales se tiene acceso a un ambiente similar al provisto en la red principal Ethereum, pero con la ventaja que en esta no se incurre en ningún costo de por medio, ya que dichas plataformas proveen de un número de ethers cada cierto tiempo facilitando así el despliegue y operación de los contratos

Finalmente, una vez ya desplegada la solución se debe realizar un mantenimiento a la misma con el objetivo de evitar fallas y vulneraciones. La fase de mantenimiento también da lugar a posibles actualizaciones de los contratos inteligentes según sea la situación de la cadena agrícola.

\section{Resultados}

Como resultados de la investigación se logró establecer la solución blockchain que puede observarse en la figura 5 en la que se detalla en el literal a, la consulta de un producto que ha pasado por todas las fases de producción. Mientras que en el literal b se puede observar un producto que está 
iniciando el proceso productivo, se debe destacar que esta información visualizada proviene de blockchain, y puede ser consultada además desde EtherScan, el cual es un buscador de transacciones y permite consultar las transacciones que han sido generadas desde una cuenta específica, o las transacciones asociadas a un contrato inteligente especifico.

a) Registro de todas las fases realizado
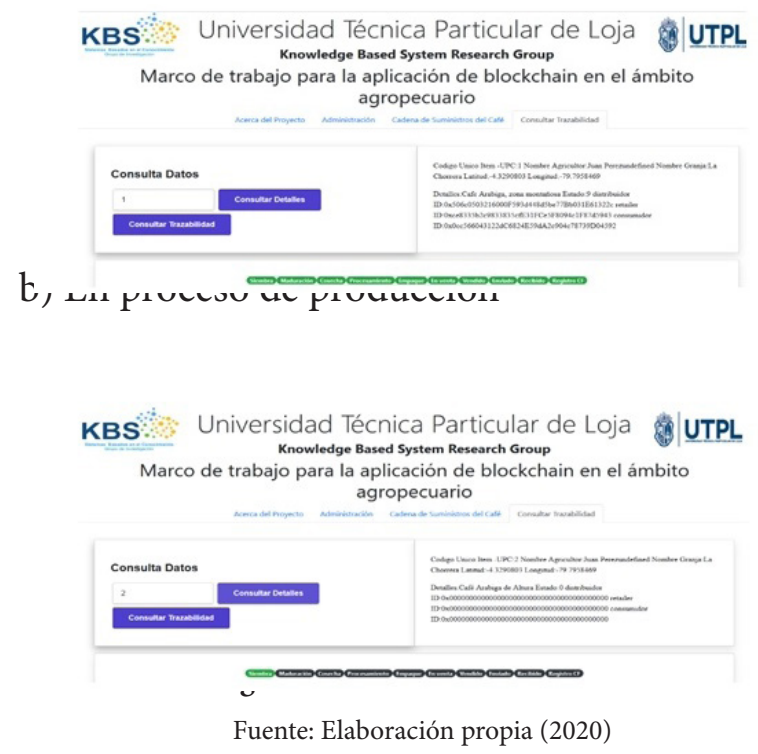

También, se puede destacar que mediante la metodología propuesta en la presente investigación es posible el desarrollo exitoso de soluciones blockchain, porque concentra distintos aspectos generales que hacen posible su adaptación para cualquier industria agrícola, alimenticia, u de otro ámbito, sin distinción de la aplicación que se desee desarrollar.

\section{Conclusiones}

Los resultados logrados del desarrollo del prototipo demuestran que es posible la gestión de una cadena de valor Agrícola empleando tecnología blockchain, además de constatar los beneficios que trae consigo la aplicación de esta tecnología en esta industria; entre estos la transparencia e inmutabilidad, por lo que se concluye que la aplicación de la tecnología blockchain en el ámbito Agrícola y especialmente enfocada en la gestión de cadenas de valor de este sector resulta muy efectivo y beneficioso, al poder lograr descentralizar y transparentar la información que muchos casos reposa en registros físicos, significando esto un gran avance en la digitalización de la información de este sector, lo cual en los últimos años se ha convertido de gran interés para este ámbito.

La integración de tecnología en el campo Agrícola permitiría lograr la transparencia del proceso productivo y así generar mayor confianza en este sector. Existen varias alternativas a nivel tecnológico para implementar soluciones blockchain, sin embargo, es necesario comprender el proceso productivo, y otros aspectos como son la conectividad, cultura, entre otros.

En la situación local y latinoamericana se ha concluido que el principal desafío para implantar una solución basada en blockchain que permita la transparencia y trazabilidad del proceso productivo, sería el compromiso que deben adquirir todos los actores que intervienen en la producción del proceso.

Otro desafío observado en el contexto es la conectividad y acceso a la tecnología de parte de algunos actores al considerar la situación geográfica en la que se encuentran, lo cual ocurre debido a muchos factores.

Existen variedad de plataformas blockchain, sin embargo, Ethereum es la plataforma que presenta mayor madurez y constante evolución; además, de disponer de diversos servicios cloud accesibles, tanto para el establecimiento de un nodo, como para la prueba de contratos inteligentes.

Se recomienda realizar un análisis de las necesidades previas a la selección de la plataforma blockchain, es decir, verificar aspectos relacionados con la confidencialidad que se desea, dispositivos desde los que se van a usar, entre otros, puesto que estos aspectos definirán el tipo de blockchain requerido, compatibilidad con frameworks, los requerimientos a nivel técnico y de infraestructura. 


\section{Referencias Bibliográficas}

Arena, A., Bianchini, A., Perazzo, P., Vallati, C., \& Dini, G. (2019). BRUSCHETTA: An IoT Blockchain-Based Framework for Certifying Extra Virgin Olive Oil Supply Chain. 173-179.

https://doi.org/10.1109/SMARTCOMP.2019.00049

Baralla, G., Pinna, A., \& Corrias, G. (2019). Ensure Traceability in European Food Supply Chain by Using a Blockchain System. 2019 IEEE/ACM 2nd International Workshop on Emerging Trends in Software Engineering for Blockchain (WETSEB), 40-47. https://doi.org/10.1109/ WETSEB.2019.00012

Borah, M. D., Naik, V. B., Patgiri, R., Bhargav, A., Phukan, B., \& Basani, S. G. M. (2020). Supply Chain Management in Agriculture Using Blockchain and IoT. Springer Singapore. https://doi.org/10.1007/978-981-13-8775-3_11

Bordel, B., Lebigot, P., Alcarria, R., \& Robles, T. (2018). Digital Food Product Traceability: Using Blockchain in the International Commerce. The 2018 International Conference on Digital Science, 224-231.

https://doi.org/10.1007/978-3-030-02351-5_27

Borrero, J. D. (2019). Sistema de trazabilidad de la cadena de suministro agroalimentario para cooperativas de frutas y hortalizas basado en la tecnología Blockchain. CIRIEC-España, Revista de Economía Pública, Social y Cooperativa, 95, 71. https://doi.org/10.7203/CIRIEC-E.95.13123

Branco, F., Moreira, F., Martins, J., Au-Yong-Oliveira, M., \& Gonçalves, R. (2019). Conceptual Approach for an Extension to a Mushroom Farm Distributed Process Control System: IoT and Blockchain. World Conference on Information Systems and Technologies, 738-747.

https://doi.org/10.1007/978-3-030-16181-1_69

Caro, M. P., Ali, M. S., Vecchio, M., \& Giaffreda, R. (2018). Blockchain-based traceability in Agri-Food supply chain management: A practical implementation. 2018 IoT Vertical and Topical Summit on Agriculture - Tuscany, IOT Tuscany 2018, 1-4.

https://doi.org/10.1109/IOT-TUSCANY.2018.8373021

Dhillon, V., Metcalf, D., \& Hooper, M. (2017). Blockchain Enabled Applications. Apress.

https://doi.org/10.1007/978-1-4842-3081-7

Food and Agriculture Organization of the United Nations. (2019). E-Agriculture in action: Blockchain for Agriculture. Opportunities and Challenges (G. Sylvester (ed.)). FAO.

Food and Agriculture Organization of the United Nations. (2020). Seguridad alimentaria y nutrición para todos.

Huang, H., Zhou, X., \& Liu, J. (2019). Food Supply Chain Traceability Scheme based on Blockchain and EPC Technology. International Conference on Smart Blockchain, 32-42.

https://doi.org/10.1007/978-3-030-34083-4_4

Laurence, T. (2019). Blockchain for dummies. In Wiley Brand (Vol. 2). John Wiley \& Sons, Inc.https://doi. org/10.1192/bjp.112.483.211-a

Lin, Q., Wang, H., Pei, X., \& Wang, J. (2019). Food Safety
Traceability System Based on Blockchain and EPCIS. IEEE Access, 7(c), 20698-20707.

https://doi.org/10.1109/ACCESS.2019.2897792

Malik, S., Kanhere, S. S., \& Jurdak, R. (2018). ProductChain: Scalable blockchain framework to support provenance in supply chains. NCA 2018 - 2018 IEEE 17th International Symposium on Network Computing and Applications, 1-10.

https://doi.org/10.1109/NCA.2018.8548322

Mao, D., Hao, Z., Wang, F., \& Li, H. (2019). Novel Automatic Food Trading System Using Consortium Blockchain. Arabian Journal for Science and Engineering, 44(4), 34393455.

https://doi.org/10.1007/s13369-018-3537-z

Mosquera, J., \& Piedra, N. (2020). Marco Metódologico para la integración de la tecnología blockchain en la industria del café. Applications in Software Engineering - Proceedings of the 9th International Conference on Software Process Improvement, CIMPS 2020.

Okoli, C., \& Schabram, K. (2010). A Guide to Conducting a Systematic Literature Review of Information Systems Research. Working Papers on Information Systems, 10(2010). https://doi.org/10.2139/ssrn.1954824

Piedra, N., \& Mosquera Arévalo, J. P. (2020). Linked Data and Musical Information to Improvement the Cultural and Heritage Knowledge Management (pp. 141-155).

https://doi.org/10.1007/978-3-030-33547-2_12

Ponomarev, E. (2020). Decentralized Web Developer Report 2020. Medium.

Prieto, J., \& Ferretti, S. (2020). Blockchain and Applications.

https://doi.org/10.1007/978-3-030-23813-1

Salah, K., Nizamuddin, N., Jayaraman, R., \& Omar, M. (2019). Blockchain-Based Soybean Traceability in Agricultural Supply Chain. IEEE Access, 7, 73295-73305.

https://doi.org/10.1109/ACCESS.2019.2918000

Takyar, A. (2020). Top Blockchain Platforms of 2020.

Viere, T., von Enden, J., \& Schaltegger, S. (2011). Life Cycle and Supply Chain Information in Environmental Management Accounting: A Coffee Case Study. 23-40. https://doi. org/10.1007/978-94-007-1390-1_2 